\title{
REVIEW
}

Open Access

\section{Candida thrombophlebitis in children: a systematic review of the literature}

Claudia Colomba', Luigi Campa', Lucia Siracusa², Salvatore Giordano², Maria C. Vella', Giovanni Corsello', Mario Giuffrè ${ }^{1 *}$ (iD and Antonio Cascio ${ }^{1}$

\begin{abstract}
Objective: To describe a case of thrombophlebitis associated with Candida infection and to analyze other published reports to define clinical characteristics, prognostic data, diagnostic and therapeutic strategies.

Study design: A computerized search was performed without language restriction using PubMed and Scopus databases. An article was considered eligible for inclusion if it reported cases with Candida thrombophlebitis. Our case was also included in the analysis.

Results: A total of 16 articles reporting 27 cases of Candida thrombophlebitis were included in our review. The median age of patients was 4 years. In 10 cases there was a thrombophlebitis of peripheral veins; in the remaining cases the deep venous circle was interested. Candida albicans was the most frequently involved fungal species. The most recurrent risk factors were central venous catheter (19/28), broad spectrum antibiotics (17/28), intensive care unit (8/28), surgery (3/28), mechanical assisted ventilation (5/28), total parenteral nutrition (8/28), cancer (2/28), premature birth (6/28), cystic fibrosis (2/28). Fever was the most frequent clinical feature. All children with peripheral and deep thrombophlebitis were given antifungal therapy: amphotericin B was the most used, alone or in combination with other antifungal drugs. Heparin was most frequently used as anticoagulant therapy. Illness was fatal in two cases.
\end{abstract}

Conclusion: Candida thrombophlebitis is a rare but likely underdiagnosed infectious complication in pediatric critically ill patients. It is closely connected to risk factors such as central venous catheter, hospitalization in intensive care unit, prematurity, assisted ventilation, chronic inflammatory diseases. Antifungal therapy and anticoagulant drugs should be optimized for each patient and surgical resection is considered in the persistence of illness.

Keywords: Thrombosis, Phlebitis, Children, Newborn, Candida, Sepsis, Hospital-acquired infections, Persistent fever, Enoxaparin, Antifungal therapy

\footnotetext{
* Correspondence: mario.giuffre@unipa.it

'Department of Health Promotion, Maternal and Infant Care, Internal Medicine and Medical Specialties, University of Palermo, Palermo, Italy

Full list of author information is available at the end of the article
}

(C) The Author(s). 2020 Open Access This article is licensed under a Creative Commons Attribution 4.0 International License, which permits use, sharing, adaptation, distribution and reproduction in any medium or format, as long as you give appropriate credit to the original author(s) and the source, provide a link to the Creative Commons licence, and indicate if changes were made. The images or other third party material in this article are included in the article's Creative Commons licence, unless indicated otherwise in a credit line to the material. If material is not included in the article's Creative Commons licence and your intended use is not permitted by statutory regulation or exceeds the permitted use, you will need to obtain permission directly from the copyright holder. To view a copy of this licence, visit http://creativecommons.org/licenses/by/4.0/ The Creative Commons Public Domain Dedication waiver (http://creativecommons.org/publicdomain/zero/1.0/) applies to the data made available in this article, unless otherwise stated in a credit line to the data. 


\section{Introduction}

Suppurative thrombophlebitis complicating intravenous therapy is a rare but important health-care associated infection. It is well described in adult patients but unfrequently reported in children [1]. It has most often been associated with Staphylococcus aureus or coagulasenegative Staphylococci, even if the incidence of Candida infections is increasing [2]. Candida thrombophlebitis can affect the superficial or deep venous circulation. The latter is an even rarer event, reported more often in adult people. Central venous catheters (CVCs) represent an important predisposing factor, especially in combination with total parenteral nutrition (TPN) and multiple antibiotic therapy [3]. Clinical signs suggestive of suppurative peripheral thrombophlebitis are persisting inflammatory signs at a previous site of an intravenous catheter insertion, complicated by systemic symptoms including high fever and malaise. Instead, diagnosis of deep Candida thrombosis may be difficult and there must be a very high level of awareness whenever a patient has the risk factors for Candida septic thrombophlebitis [1, 3].

Peripheral thrombophlebitis often responds well to catheter removal, local drainage, short course of antifungal therapy and surgical excision of the thrombosed part of the vein is recommended [4].

Central venous infection poses a greater problem, however, due to the formidable undertaking in surgical remove of the clot from the central veins and thrombophlebitis can be successfully managed by removal of the catheter, antifungal therapy, and systemic anticoagulation therapy $[3,4]$.

\section{We describe here the case of a child who developed thrombophlebitis of the iliac-femoral axis by Candida albicans}

The aim of our study is to focus attention on Candida thrombophlebitis in children and to analyze the epidemiologic and clinical characteristics of Candida thrombophlebitis in pediatric patients through a systematic review of the literature.

\section{Case report}

A 2-year-old girl with a recent history of recurrent respiratory infections was admitted to the "G. Di Cristina" Children's Hospital in Palermo, Sicily, Italy, for fever and cough. Upon admission, she had mild dyspnea with polypnea (respiratory rate $40 / \mathrm{min}$ ), with bilateral basal crackles in both lungs. Blood tests revealed white blood cell $9.810 / \mathrm{mm}^{3}$ (neutrophils $40 \%$, leukocytes 55\%) and C-reactive protein $2,66 \mathrm{mg} / \mathrm{dl}$. A chest X-ray revealed pneumonia. Treatment with ceftazidime $(100 \mathrm{mg} / \mathrm{kg} / \mathrm{die})$ was started with resolution of fever after 3 days. Ten days later, she presented fever and clinical worsening, respiratory distress, onset of pneumothorax and subcutaneous emphysema involving the upper limb and neck, the patient was transferred to the ICU and a central venous catheter was placed in the left femoral vein. Seven days after, the patient developed continuous fever (higher temperature $40{ }^{\circ} \mathrm{C}$ ) and CVC was removed. Blood and CVC cultures were positive for azole-resistant Candida albicans. The patient was transferred to the Infectious Disease Unit where a treatment with amphotericin $\mathrm{B}(3 \mathrm{mg} / \mathrm{kg} / \mathrm{die})$ and caspofungin $\left(70 \mathrm{mg} / \mathrm{m}^{2} /\right.$ die in first day, then $50 \mathrm{mg} / \mathrm{m}^{2} / \mathrm{die}$ ) was started. For the persistence of fever and onset of edema of the lower limb, a chest and abdominal CT scan was performed with evidence of thrombophlebitis of left iliac-femoral axis and associated fistula of paravertebral and gluteal muscles, confirmed by a subsequent thorax and abdomen MRI scan. Therapy with sub cutaneous enoxaparin was started $(100 \mathrm{UI} / \mathrm{kg})$. A sweat test was carried out, with positive result, but subsequent genetic study excluded mutations associated to cystic fibrosis.

After a week, fever persisted and a CT scan showed no improvement of the deep vein thrombosis extension. The child was transferred to another hospital unit, specialized in thrombosis in pediatric age, where treatment was implemented with prednisone administration. A gradual improvement of clinical and instrumental picture was achieved without surgical resection.

\section{Literature search}

A computerized search was performed without language restriction using PubMed and Scopus, combining the terms (phlebitis OR venous thrombosis OR thrombosis) AND (candid* OR fungal*) AND (children OR child OR infant OR baby), with no filters. Furthermore, all references listed were hand-searched for other relevant articles. An article was considered eligible for inclusion if it reported cases with full clinical data consistent with Candida thrombophlebitis. The following epidemiologic and clinical variables were evaluated for each case: sex, age, risk factors for candidemia, Candida species, clinical features, therapy and outcome.

The selected articles were reviewed by two independent authors, and judged on their relevant contribution to the subject of the study. The Preferred Reporting Items for Systematic Review And Meta-Analysis (PRISMA) guidelines were followed.

\section{Results}

After an extensive search in MEDLINE, 355 articles were found; only 13 of the above papers were selected for inclusion [5-17]. Three additional papers not present in Medline were added after a hand search of the bibliography of the above papers [18-20]. 
A total of 16 articles reporting 27 cases of Candida thrombophlebitis were included in our review. A flow chart summarizing the literature research approach is reported in Fig. 1. Most of the articles were single case reports.

We analyzed anamnestic and clinical data of 28 patients (including our new case). Risk factors, clinical features, diagnosis, therapy and mortality have been analytically reported in Table 1 .

There were 7 boys and 9 girls, in the remaining cases the gender was not specified. The median age was 4 years. Six cases were reported in premature newborns. Fourteen children were under 24 months old. In 10 cases thrombophlebitis interested peripheral veins $[8,18,20]$, in the remaining cases the deep venous circle.

Candida albicans was isolated in 18 cases, Candida parapsilosis in 9 and Candida tropicalis in 4. In two cases 2 different species were simultaneously present (in one case C. albicans and C. parapsilosis, in the other case C. albicans and C. tropicalis). Risk factors for candidemia and thrombosis included CVC (19/28, 67\%), broad spectrum antibiotics $(17 / 28,60 \%)$, admission to the $\operatorname{ICU}(8 / 28,28 \%)$, surgery $(3 / 28,10 \%)$, mechanical assisted ventilation $(5 / 28,17 \%)$, TPN $(8 / 28,28 \%)$, cancer $(2 / 28,7 \%)$, premature birth $(6 / 28,21 \%)$, cystic fibrosis
$(2 / 28,7 \%)$. In all cases of peripheral thrombophlebitis, the thrombosed and infected veins appeared thickened with inflammation of the overlying soft tissue and fever was present in 3 cases $[12,18,20]$. Among patients with deep venous thrombophlebitis, $88 \%$ patients $(16 / 18)$ were febrile at the time of diagnosis.

Caval thrombosis was suspected in most patients on the basis of head and neck swelling. In all cases diagnosis was highly suspected because of persistence of candidemia and a vascular catheter in place prior to the onset of candidemia. Diagnosis was confirmed with wholebody gallium 67 scintigraphy in 2 cases, with venogram in 3 cases, with echocardiogram in 4 cases, and with ultrasonography in 9 cases.

All children with peripheral and deep thrombophlebitis were given antifungal therapy: 19 patients were treated with amphotericin B, 11 of which with a combination of amphotericin B and 5-fluorocytosine, 2 with amphotericin B and echinocandin, one with amphotericin B and fluconazole and 5 with amphotericin B as monotherapy. Fluconazole has been used successfully on monotherapy in 6 cases. Liposomal amphotericin B was used only in another case apart from ours [14]. In 3/10 cases of the superficial thrombophlebitis, excision of the thrombosed part of the vein was necessary $[8,18,20]$

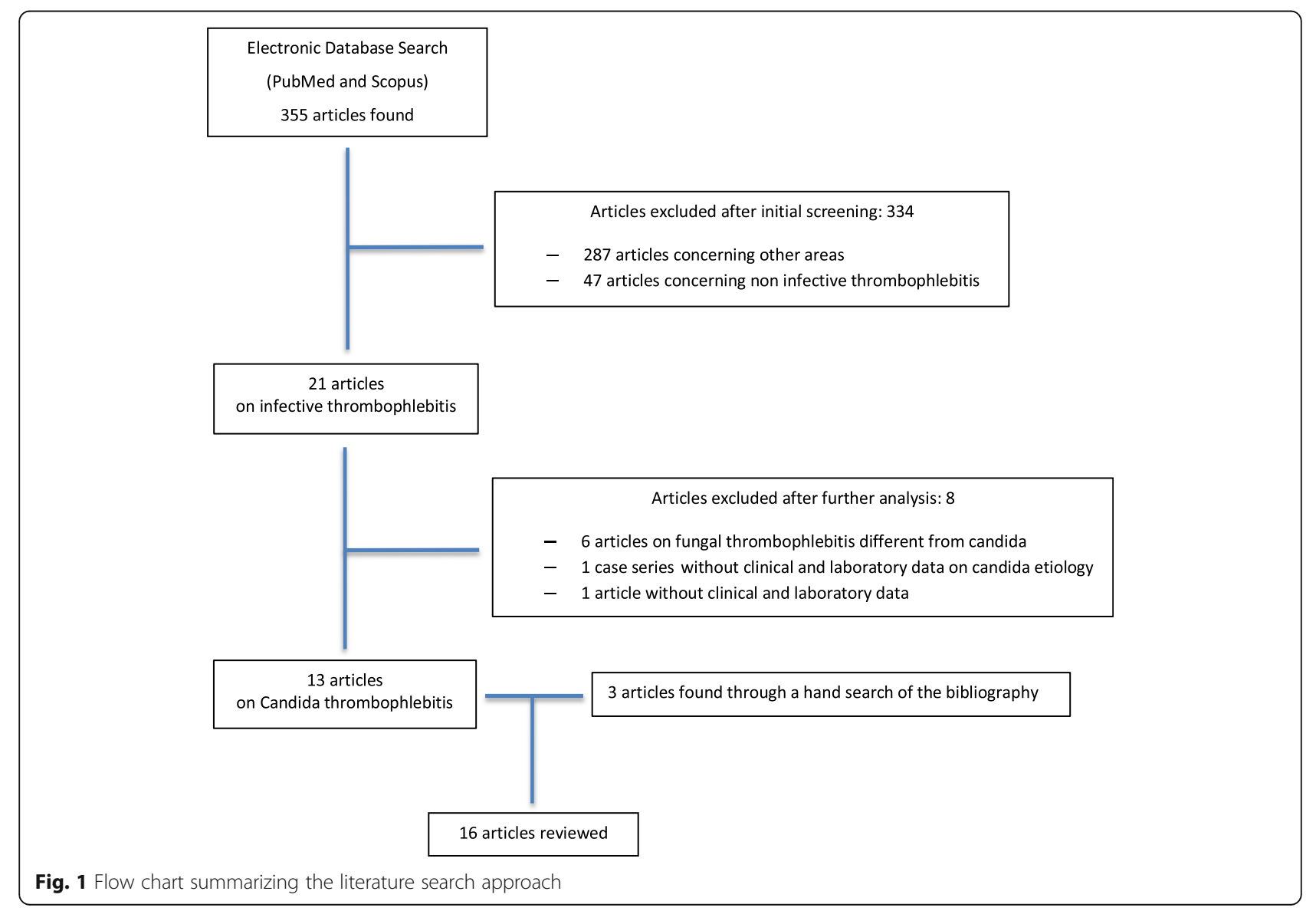


Table 1 Data from the reported cases of Candida thrombophlebitis in pediatric patients

\begin{tabular}{|c|c|c|c|c|c|c|c|c|}
\hline $\begin{array}{l}\text { Author/ } \\
\text { year/ } \\
\text { Country } \\
\text { [Ref] }\end{array}$ & $\begin{array}{l}\text { Age/ } \\
\text { Sex }\end{array}$ & $\begin{array}{l}\text { Initial clinical } \\
\text { conditions and/or } \\
\text { related procedures }\end{array}$ & Risk factors & $\begin{array}{l}\text { Candida } \\
\text { species }\end{array}$ & Location & Presentation & Therapy & Outcome \\
\hline $\begin{array}{l}\text { Wiley/ } \\
\text { 1977/USA } \\
\text { [5] }\end{array}$ & $\begin{array}{l}\mathrm{PN} / \\
\mathrm{F}\end{array}$ & $\begin{array}{l}\text { tracheoesophageal } \\
\text { fistula, gastrostomy, } \\
\text { cardiopulmunary } \\
\text { arrests, aspiration } \\
\text { pneumonia }\end{array}$ & CVC, TPN, ICU, surgery, AB & $\begin{array}{l}\text { C. albicans } \\
\text { C. } \\
\text { parapsilosis }\end{array}$ & SVC, RV & $\begin{array}{l}\text { edema of the } \\
\text { face and } \\
\text { neck }\end{array}$ & $\begin{array}{l}\text { amfotericin B5, } \\
\text { flucytosine }\end{array}$ & died \\
\hline \multirow[t]{2}{*}{$\begin{array}{l}\text { Miller/ } \\
\text { 1981/USA } \\
{[6]}\end{array}$} & $\begin{array}{l}18 \\
\text { mo / } \\
M\end{array}$ & $\begin{array}{l}\text { acute } \\
\text { undifferentiated } \\
\text { leukemia }\end{array}$ & CVC, cancer, $C T, A B$ & C. albicans & $\begin{array}{l}\text { axillary and } \\
\text { subclavian } \\
\text { vein }\end{array}$ & fever & $\begin{array}{l}\text { antifungal } \\
\text { therapy (n.s.) }\end{array}$ & survived \\
\hline & $\begin{array}{l}10 y \\
/ F\end{array}$ & $\begin{array}{l}\text { acute myeloid } \\
\text { leukemia }\end{array}$ & CVC, cancer & C. tropicalis & giugular vein & fever & $\begin{array}{l}\text { antinfungal } \\
\text { agents (n.s) }\end{array}$ & survived \\
\hline $\begin{array}{l}\text { Torres- } \\
\text { Rojas/ } \\
\text { 1982/USA } \\
{[18]}\end{array}$ & $\begin{array}{l}11 y \\
/ F\end{array}$ & acute rheumatic fever & $A B$, steroids & C. albicans. & n.s. & fever & $\begin{array}{l}\text { amphotericin B, } \\
\text { 5-fluorocytosine, } \\
\text { excision of vein }\end{array}$ & survived \\
\hline $\begin{array}{l}\text { Malfroot/ } \\
\text { 1986/ } \\
\text { Belgium } \\
\text { [20] }\end{array}$ & $\begin{array}{l}17 y \\
/ M\end{array}$ & CF, pneumonia & $\begin{array}{l}\mathrm{AB}, \mathrm{CVC} \text {, advanced pulmonary } \\
\text { disease }\end{array}$ & C. albicans & basilica vein & $\begin{array}{l}\text { fever, } \\
\text { phlebitis }\end{array}$ & $\begin{array}{l}\text { amphotericin B, } \\
\text { flucytosine, } \\
\text { surgical excision }\end{array}$ & survived \\
\hline \multirow[t]{4}{*}{$\begin{array}{l}\text { Lacey/ } \\
\text { 1988/USA } \\
{[7]}\end{array}$} & $\begin{array}{l}13 \\
\mathrm{mo} / \\
\mathrm{NR}\end{array}$ & $\begin{array}{l}\text { broncopulmonary } \\
\text { displasia, ECMO } \\
\text { survivor }\end{array}$ & $A B, T P N, C V C$ & C. albicans & IVC & $\begin{array}{l}\text { sepsis, head } \\
\text { and neck } \\
\text { swelling }\end{array}$ & $\begin{array}{l}\text { amphotericin B, } \\
5 \text { fluorocytosine, } \\
\text { streptokinase }\end{array}$ & survived \\
\hline & $\begin{array}{l}4 \\
\text { mo / } \\
\text { NR }\end{array}$ & midgut volvulus & $A B, T P N, C V C$ & $\begin{array}{l}\text { C. } \\
\text { tropicalis }\end{array}$ & SVC, IVC & $\begin{array}{l}\text { sepsis, head } \\
\text { and neck } \\
\text { swelling }\end{array}$ & $\begin{array}{l}\text { amphotericin B } \\
5 \text { fluorocytosine } \\
\text { streptokinase }\end{array}$ & survived \\
\hline & $\begin{array}{l}4 \\
\text { mo / } \\
\text { NR }\end{array}$ & pentalogy of Cantrell & $A B, T P N, C V C$ & C. albicans & SVC & $\begin{array}{l}\text { sepsis, head } \\
\text { and neck } \\
\text { swelling }\end{array}$ & $\begin{array}{l}\text { amphotericin-B, } \\
5 \text { fluorocytosine, } \\
\text { streptokinase }\end{array}$ & survived \\
\hline & $\begin{array}{l}11 \\
\text { mo / } \\
\text { NR }\end{array}$ & $\begin{array}{l}\text { Hirschsprung's } \\
\text { disease }\end{array}$ & $A B, T P N, C V C$ & C. albicans & SVC, IVC & $\begin{array}{l}\text { sepsis, head } \\
\text { and neck } \\
\text { swelling }\end{array}$ & $\begin{array}{l}\text { amphotericin B, } \\
5 \text { fluorocytosine, } \\
\text { streptokinase }\end{array}$ & survived \\
\hline $\begin{array}{l}\text { Cotton/ } \\
\text { 1988/Rep. } \\
\text { of South } \\
\text { Africa [8] }\end{array}$ & $\begin{array}{l}\text { PN / } \\
\text { F }\end{array}$ & $\begin{array}{l}\text { hyaline membrane } \\
\text { disease }\end{array}$ & assisted ventilation, $A B$ & $\begin{array}{l}\text { C. } \\
\text { parapsilosis }\end{array}$ & $\begin{array}{l}\text { vein of the } \\
\text { dorsum of the } \\
\text { foot }\end{array}$ & phlebitis & $\begin{array}{l}\text { amphotericin } B \text {, } \\
\text { surgical excision }\end{array}$ & survived \\
\hline $\begin{array}{l}\text { Berg/1989/ } \\
\text { USA [9] }\end{array}$ & $\begin{array}{l}5 y / \\
F\end{array}$ & $\begin{array}{l}\text { burns and surgical } \\
\text { debridement, sepsis, } \\
\text { pneumonia }\end{array}$ & wound infection, ICU, AB, CVC & C. albicans & $\begin{array}{l}\text { iliac and } \\
\text { femoral veins, } \\
\text { IVC }\end{array}$ & $\begin{array}{l}\text { sepsis, } \\
\text { phlebitis }\end{array}$ & $\begin{array}{l}\text { amphotericinB, } \\
\text { flucytosine, } \\
\text { heparin }\end{array}$ & survived \\
\hline $\begin{array}{l}\text { Ashkenazi/ } \\
\text { 1990/USA } \\
\text { [10] }\end{array}$ & $\begin{array}{l}4 \\
\mathrm{mo} / \\
\mathrm{M}\end{array}$ & $\begin{array}{l}\text { upper respiratory } \\
\text { tract infection, } \\
\text { respiratory distress } \\
\text { (by RSV), double } \\
\text { aortic arch }\end{array}$ & $C V C$, surgery, ICU, AB & $\begin{array}{l}\text { C. } \\
\text { tropicalis }\end{array}$ & IVC, renal vein & fever & $\begin{array}{l}\text { amphotericin } \mathrm{B} \text {, } \\
\text { heparin }\end{array}$ & survived \\
\hline $\begin{array}{l}\text { Cherrick/ } \\
\text { 1995/USA } \\
\text { [11] }\end{array}$ & $\begin{array}{l}16 \\
\mathrm{mo} / \\
\mathrm{M}\end{array}$ & neuroblastoma, sepsis & $C V C, A B, T P N, C T$ & C. albicans & $\begin{array}{l}\text { innominate } \\
\text { vein }\end{array}$ & $\begin{array}{l}\text { fever, } \\
\text { respiratory } \\
\text { distress }\end{array}$ & $\begin{array}{l}\text { amphotericin B, } \\
\text { flucytosine, } \\
\text { streptokinase }\end{array}$ & survived \\
\hline \multirow{4}{*}{$\begin{array}{l}\text { Friedland/ } \\
\text { 1996/Rep. } \\
\text { of South } \\
\text { Africa [12] }\end{array}$} & $\begin{array}{l}6 \\
\text { mo / } \\
\text { NR }\end{array}$ & diarrhea, sepsis & n.s. & $\begin{array}{l}\text { C. } \\
\text { parapsilosis }\end{array}$ & cubital vein & fever & fluconazole & survived \\
\hline & $\begin{array}{l}3 \\
\text { mo / } \\
\text { NR }\end{array}$ & diarrhea & malnutrition & $\begin{array}{l}\text { C. } \\
\text { parapsilosis }\end{array}$ & cubital vein & phlebitis & $\begin{array}{l}\text { flucanazole, } \\
\text { incision and } \\
\text { drainage }\end{array}$ & survived \\
\hline & $\begin{array}{l}7 \\
\text { mo / } \\
\text { NR }\end{array}$ & pneumonia & n.s. & C. albicans & forearm vein & phlebitis & fluconazole & survived \\
\hline & $\begin{array}{l}22 \\
\mathrm{mo} /\end{array}$ & prematurity & prematurity & C. albicans & cubital & phlebitis & $\begin{array}{l}\text { fluconazole, } \\
\text { incision and }\end{array}$ & survived \\
\hline
\end{tabular}


Table 1 Data from the reported cases of Candida thrombophlebitis in pediatric patients (Continued)

\begin{tabular}{|c|c|c|c|c|c|c|c|c|}
\hline $\begin{array}{l}\text { Author/ } \\
\text { year/ } \\
\text { Country } \\
\text { [Ref] }\end{array}$ & $\begin{array}{l}\text { Age/ } \\
\text { Sex }\end{array}$ & $\begin{array}{l}\text { Initial clinical } \\
\text { conditions and/or } \\
\text { related procedures }\end{array}$ & Risk factors & $\begin{array}{l}\text { Candida } \\
\text { species }\end{array}$ & Location & Presentation & Therapy & Outcome \\
\hline & $N R$ & & & & & & drainage & \\
\hline & $\begin{array}{l}4 \\
\text { mo / } \\
\text { NR }\end{array}$ & diarrhea & n.s. & $\begin{array}{l}\text { C. } \\
\text { parapsilosis }\end{array}$ & hand vein & phlebitis & $\begin{array}{l}\text { fluconazole, } \\
\text { aspiration }\end{array}$ & survived \\
\hline & $\begin{array}{l}8 \\
\text { mo / } \\
\text { NR }\end{array}$ & diarrhea, HIV & HIV & $\begin{array}{l}\text { C. } \\
\text { parapsilosis }\end{array}$ & cubital vein & phlebitis & $\begin{array}{l}\text { amphotericin B, } \\
\text { aspiration }\end{array}$ & survived \\
\hline & $\begin{array}{l}9 \\
\text { mo / } \\
\text { NR }\end{array}$ & diarrhea & TPN & $\begin{array}{l}\text { C. } \\
\text { parapsilosis }\end{array}$ & cubital vein & phlebitis & $\begin{array}{l}\text { amphotericin B, } \\
\text { incision and } \\
\text { drainage }\end{array}$ & survived \\
\hline $\begin{array}{l}\text { Garcia/ } \\
\text { 1997/ } \\
\text { France [19] }\end{array}$ & $\begin{array}{l}14 y \\
/ F\end{array}$ & $\begin{array}{l}\text { ventricular } \\
\text { tachycardia, cardiac } \\
\text { arrest }\end{array}$ & ICU, CVC, MAV & C. albicans & $\begin{array}{l}\text { left subclavian } \\
\text { vein, right } \\
\text { giugula, SVC, } \\
\text { RA }\end{array}$ & fever & $\begin{array}{l}\text { amphotericin B, } \\
\text { flucytosine, } \\
\text { heparin, } \\
\text { caval } \\
\text { thrombectomy }\end{array}$ & survived \\
\hline \multirow[t]{2}{*}{$\begin{array}{l}\text { Pacifico/ } \\
\text { 2004/Italy } \\
\text { [13] }\end{array}$} & $\begin{array}{l}\mathrm{PN} / \\
\mathrm{M}\end{array}$ & $\begin{array}{l}\text { respiratory distress, } \\
\text { prematurity }\end{array}$ & $\begin{array}{l}\text { tri-twin pregnancy, } \\
\text { prematurity, CVC, NICU, AB, } \\
\text { MAV, maternal fever }\end{array}$ & C. albicans & $\begin{array}{l}\text { right and main } \\
\text { portal veins }\end{array}$ & $\begin{array}{l}\text { abdominal } \\
\text { distension }\end{array}$ & $\begin{array}{l}\text { amphotericin B, } \\
\text { fluconazole }\end{array}$ & survived \\
\hline & $\begin{array}{l}\mathrm{PN} / \\
\mathrm{M}\end{array}$ & $\begin{array}{l}\text { prematurity, maternal } \\
\text { fever }\end{array}$ & $\begin{array}{l}\text { prematurity, NICU, CVC, MAV, } \\
A B\end{array}$ & C. albicans & $\begin{array}{l}\text { main portal } \\
\text { vein }\end{array}$ & $\begin{array}{l}\text { fever, } \\
\text { abdominal } \\
\text { distension }\end{array}$ & amphotericin B & survived \\
\hline $\begin{array}{l}\text { Sharma/ } \\
\text { 2009/USA } \\
{[14]}\end{array}$ & $\begin{array}{l}\mathrm{PN} / \\
\mathrm{M}\end{array}$ & $\begin{array}{l}\text { prematurity, patent } \\
\text { ductus arteriosus, } \\
\text { respiratory distress } \\
\text { syndrome, intestinal } \\
\text { perforation }\end{array}$ & prematurity, $A B$, surgery, $C V C$ & C. albicans & RA, IVC & NR & $\begin{array}{l}\text { liposomal } \\
\text { amphotericin B, } \\
\text { caspofungin, } \\
\text { heparin }\end{array}$ & died \\
\hline $\begin{array}{l}\text { Giuffrè/ } \\
\text { 2016/Italy } \\
\text { [15] }\end{array}$ & $\begin{array}{l}\mathrm{PN} / \\
\mathrm{NR}\end{array}$ & prematurity & $\begin{array}{l}\text { prematurity, TPN, UVC, } \\
\text { intestinal occlusion, MTHFR } \\
\text { gene mutation (C677T), } \\
\text { homozygous PAI-1 gene poly- } \\
\text { morphism (4G/4G) }\end{array}$ & $\begin{array}{l}\text { C. } \\
\text { parapsilosis }\end{array}$ & $\begin{array}{l}\text { umbilical vein } \\
\text { and left } \\
\text { branch of the } \\
\text { hepatic portal } \\
\text { vein }\end{array}$ & $\begin{array}{l}\text { perihepatic } \\
\text { abscess }\end{array}$ & $\begin{array}{l}\text { drainage of the } \\
\text { collection, } \\
\text { removal of } \\
\text { umbilical } \\
\text { vessels, } \\
\text { micafungin }\end{array}$ & survived \\
\hline $\begin{array}{l}\text { Manish/ } \\
\text { 2017/India } \\
{[16]}\end{array}$ & $\begin{array}{l}4 y / \\
F\end{array}$ & diabetic ketoacidosis & ICU, MAV, CVC & $\begin{array}{l}\text { C. albicans } \\
\text { C. tropical }\end{array}$ & $\begin{array}{l}\text { right external } \\
\text { iliac vein, } \\
\text { common } \\
\text { femoral, deep } \\
\text { femoral vein }\end{array}$ & fever & fluconazole & survived \\
\hline $\begin{array}{l}\text { Schapkaitz/ } \\
\text { 2019/Rep. } \\
\text { of South } \\
\text { Africa [17] }\end{array}$ & $5 \mathrm{y} / \mathrm{F}$ & $\begin{array}{l}C F \text {, respiratory } \\
\text { infection, } A B\end{array}$ & $C V C, A B$ & $\begin{array}{l}\text { C. } \\
\text { parapsilosis }\end{array}$ & $\begin{array}{l}\text { endocarditis, } \\
\text { SVC, RA }\end{array}$ & fever & $\begin{array}{l}\text { amphotericin B, } \\
\text { fluconazole }\end{array}$ & survived \\
\hline $\begin{array}{l}\text { present } \\
\text { case/2020/ } \\
\text { Italy }\end{array}$ & $\begin{array}{l}2 y / \\
F\end{array}$ & $\begin{array}{l}\text { respiratory infection, } \\
\text { pneumothorax and } \\
\text { subcutaneous } \\
\text { emphysema }\end{array}$ & $C V C, I C U, A B$ & C. albicans & $\begin{array}{l}\text { left iliac- } \\
\text { femoral axis }\end{array}$ & $\begin{array}{l}\text { fever, edema } \\
\text { of leg }\end{array}$ & $\begin{array}{l}\text { liposomial } \\
\text { amphotericin B, } \\
\text { caspofungin, } \\
\text { enoxaparin }\end{array}$ & survived \\
\hline
\end{tabular}

CVC Central venous catheter, ICU Intensive care unit; NICU Neonatal intensive care unit, TPN total parenteral nutrition, AB Antibiotic therapy, SCV Superior vena cava, ICV Inferior vena cava, $R V$ Right ventricle

RA Right atrial, CT Chemotherapy, CF Cystic fibrosis, RSV Respiratory syncytial virus, MAV Mechanical assisted ventilation, RV Right ventricle, UVC Umbilical vein catheter, n.s. Not specified, NR Not reported, PN Preterm newborn

and only in one case of deep thrombophlebitis the thrombus was surgically removed [19].

Data regarding anticoagulant therapy reported that heparin was used in 5 cases and thrombolytic therapy with continuous infusion of low-dose of streptokinase in others 5 cases of deep thrombophlebitis.
In two cases (both premature newborns) the outcome was fatal: one died because of a superior vena cava syndrome secondary to candida thrombophlebitis, the other one died because of renal failure secondary to inferior vena cava thrombosis with right atrial mycetoma. Both diagnoses were made at autopsy: in the first patient, the 
superior vena cava appeared obstructed throughout its length by an organizing thrombus and histology showed budding yeast and pseudohyphe [5], in the second one the atrial thrombus revealed multiple hyphae mimicking a fungal ball [14].

\section{Discussion}

Only 27 cases of Candida thrombophlebitis were retrieved after our accurate search. On the basis of the increasing incidence of nosocomial candidemia in children over the past decades and the high incidence of catheter-related thrombosis [21, 22], we would have expected to find many more cases. Therefore, we think Candida thrombophlebitis is likely an underdiagnosed or underreported disorder. Our search found that all but three cases were reported in three countries: USA, South Africa and Italy. Being Candida thrombophlebitis a health-care associated infection, we would expect it is more reported in countries with the highest incidence of hospital-acquired infections [23].

The pathogenesis of Candida thrombosis and related catheter-associated candidemia is not well understood. The source of candidemia has been a matter of debate, with some suggesting a skin origin and others a gut origin. Candidemia may arise from the gastrointestinal tract and portal venous circulation and enter the systemic circulation, leading to secondary infection of the vascular catheter $[24,25]$. In this situation, the catheter is the target of fungemia rather than the source but it might continue to be the source of sustained candidemia as organisms proliferate on its surface. Vascular catheters, however, might serve as primary source of fungemia and infectious organisms may be introduced percutaneously along the catheter, or with contaminated intravenous solution [26, 27].

Certainly, the presence of a CVC is the most important risk factor for the development of deep venous thromboembolism in children. The reported incidence in pediatric patients ranges from 5 to $44 \%$ depending on age, the primary condition, and therapies received via the CVC, as well as the type and site of CVC placement [28]. Several possible mechanisms by which CVC causes thrombosis include damage to vessel wall, disrupted blood flow, infusion of substances that damage endothelial cells (in example in case of TPN), and thrombogenic catheter materials [29].

Antibiotic therapy, given in combination with TPN, is the most important risk factor for the development of candidemia, allowing fungal gut overgrowth with subsequent absorption through intact epithelium. The combination of the above risk factors, CVC, antibiotic therapy and TPN, exposes the patient to a very high risk of deep candida thrombophlebitis. All patients reported in this review had one of the described risk factors: more than $60 \%$ received antibiotic therapy for several days,
$68 \%$ had prolonged venous catheterization and $28.6 \%$ received TPN.

Prematurity is an important risk factor for Candida thrombophlebitis. Low gestational age, low birth weight, immaturity of anatomical barriers, endotracheal intubation, mechanical ventilation, TPN, central venous catheterization, broad spectrum antibiotics treatment and surgery are considered the main risk factors for Candida spp. infection at birth [29]. All cases of neonatal thrombophlebitis in this review occurred in premature babies with more than 3 risk factors among those described above. The incidence of neonatal candidemia is reported as 2-6.8\% among Very Low Birth Weight infants (less than $1500 \mathrm{~g}$ ) and even higher in Extremely Low Birth Weight infants (less than $1000 \mathrm{~g}$ ), ranging from 4 to $16 \%$. The incidence increases with reduction of gestational age (inverse linear pattern) from around $3 \%$ at 28 weeks of gestation to $24 \%$ at 23 weeks of gestation [30, 31].

Transmission of Candida may be vertical (from the mother) or nosocomial, and umbilical vein catheter (UVC) is probably the most important risk factors. UVC positioning is a relatively easy procedure performed routinely in Neonatal Intensive Care Units. However, the possibility of an incorrect placement of the catheter may lead to a higher risk of complications, including thromboembolic complications and liver abscesses formation. The presence of a perihepatic abscess originated from a septic thrombus of the umbilical vein has been reported in literature and in our review $[15,31]$.

Our patient was investigated for cystic fibrosis, which is associated to a high frequency of acquired protein $\mathrm{C}$ and $\mathrm{S}$ deficiency as a result of vitamin $\mathrm{K}$ deficiency or associated liver involvement. Therefore, cystic fibrosis is a predisposing condition for venous thromboembolism, as occurred in 2 patients reported in this review $[17,20]$.

Deep candida thrombophlebitis is often occult and underdiagnosed; the diagnosis may be difficult, especially in the absence of metastatic phenomena [32] or localized edema and there must be a very high level of suspicion for the presence of risk factors. In our review, only patients with caval thrombosis showed head and neck swelling; in all other patients $(61 \%)$ the occurrence or persistence of fever and a persistent candidemia after removal of a culture-positive CVC, despite effective antifungal treatment, suggested the presence of an intravascular focus of infection.

We suggest the opportunity of a routine check for deep thrombophlebitis on the basis of a poor catheter function in all children with chronic indwelling CVCs and septic signs. Eventually, echo-doppler and/or CTscan of the central veins together with echocardiography should be considered for corroborating the definite diagnosis of deep thrombophlebitis [3]. 
Although radical excision of the affected veins in combination with antifungal therapy is the current recommendation in the treatment of peripheral Candida thrombophlebitis [4], in our review the excision of the thrombosed part of the vein was necessary only in 3 cases.

Few data are available concerning the best strategy for managing septic deep thrombophlebitis $[1,4]$. In some cases, systemic anticoagulation or thrombolytic therapy has been used as adjunctive therapy, but there are insufficient data to recommend their use [4]. Removal of CVC, antifungal therapy and radiologic monitoring of the thrombus are recommended [4]. Unfortunately, the decision of removing a CVC in children is more difficult than in adults, due to the frequently limited pediatric intravascular access and to the risk of thrombus dislodgement and pulmonary embolization. Regarding antifungal therapy, lipid formulation of amphotericin B (L-AmB) (3-5 mg/kg daily), or fluconazole $(6-12 \mathrm{mg} / \mathrm{kg}$ daily), or an echinocandin (caspofungin $70 \mathrm{mg} / \mathrm{m}^{2}$ as loading dose, then $50 \mathrm{mg} / \mathrm{m}^{2}$ daily, micafungin $2 \mathrm{mg} / \mathrm{kg}$ daily, or anidulafungin $1.5 \mathrm{mg} / \mathrm{kg}$ ) for at least 2 weeks after candidemia (if present) has cleared is recommended for the treatment of Candida thrombophlebitis [4]. In our review amphotericin B deoxycholate (in combination with 5-fluorocytosine in more than half of the cases) was the most used drug. Fluconazole and caspofungin have also been successfully used in some cases. In particular conditions, such as in case of kidney failure, dosages of antifungal drugs should be modified according to the plasmatic levels of creatinine. Dosage interval of fluconazole may be extended up to $48-72 \mathrm{~h}$ also in neonatal age, depending on degree of prematurity. Among echinocandins, micafungin is registered for use in the first month of life (recommended dosage $7-10 \mathrm{mg} / \mathrm{kg} / \mathrm{die}$ ) and appears to be effective also against biofilm on intravascular catheters.

Sometimes medical therapy fails because the antifungal agents are unable to reach the infected site, as occurs with an abscess during an endovascular infection. Therefore, excision of the infected vein should be important but this approach is frequently not practical for most pediatric patients with deep Candida thrombophlebitis, due to the relative inaccessibility of the central veins and the high mortality and morbidity associated with surgical thrombectomy in these critically ill infants [3]. In our review a prolonged antifungal therapy and the removal of CVC, with or without anticoagulation, resulted in clinical cure in most cases.

In conclusion Candida thrombophlebitis is a rare but likely underdiagnosed infectious complication of the critically ill patients. Recurrent or persistent candidemia after removal of a contaminated CVC should raise the suspicion leading to key investigations for an early diagnosis. Prolonged fungicidal therapy with amphotericin B or echinocandins must be provided. Treatment should be optimized for each patient and surgical resection evaluated on the basis of perioperative risk.
Abbreviations

CVC: Central venous catheter; ICU: Intensive care unit; TPN: Total parenteral nutrition

\section{Acknowledgements}

Not applicable.

\section{Authors' contributions}

CC and AC designed and wrote the paper. LC, LS and MCV collected clinical data and performed literature search. SG revised the pediatric literature. GC revised the neonatal literature. MG analyzed clinical data and revised the manuscript. All authors read and approved the final manuscript.

\section{Funding}

No funding was granted for this research.

Availability of data and materials

The datasets used and/or analyzed during the current study are available from the corresponding author on reasonable request.

Ethics approval and consent to participate

Parent's informed written consent was provided.

Consent for publication

Parent's informed written consent was provided.

\section{Competing interests}

The authors declare that they have no competing interests.

\section{Author details}

${ }^{1}$ Department of Health Promotion, Maternal and Infant Care, Internal Medicine and Medical Specialties, University of Palermo, Palermo, Italy. ${ }^{2}$ Pediatric Infectious Diseases Unit, "G. Di Cristina" Hospital, ARNAS Civico, Palermo, Italy.

Received: 24 July 2020 Accepted: 30 September 2020

Published online: 06 October 2020

\section{References}

1. Khan EA, Correa AG, Baker CJ. Suppurative thrombophlebitis in children: a ten-year experience. Pediatr Infect Dis J. 1997;16(1):63-7.

2. Leibovitz E, luster-Reicher A, Amitai M, Mogilner B. Systemic candidal infections associated with use of peripheral venous catheters in neonates: a nine-year experience. Clin Infect Dis. 1992;14(2):485-91.

3. Caccese R, Carfagna P, Pistilli N, Massetti AP, Falcone M, Venditti M. Candidal thrombophlebitis of central veins: case report and review. Med Mycol. 2012; 50(3):299-304.

4. Pappas PG, Kauffman CA, Andes DR, Clancy CJ, Marr KA, Ostrosky-Zeichner L, Reboli AC, Schuster MG, Vazquez JA, Walsh TJ, Zaoutis TE, Sobel JD. Clinical practice guideline for the management of candidiasis: 2016 update by the Infectious Diseases Society of America. Clin Infect Dis. 2016;62(4):e1-50.

5. Wiley EL, Hutchins GM. Superior vena cava syndrome secondary to Candida thrombophlebitis complicating parenteral alimentation. J Pediatr. 1977;91(6):977-9.

6. Miller JH. Detection of deep venous thrombophlebitis by gallium 67 Scintigraphy. Radiology. 1981;140(1):183-6.

7. Lacey SR, Zaritsky AL, Azizkhan RG. Successful treatment of Candida-infected Caval thrombosis in critically infants by low-dose streptokinase infusion. J Pediatr Surg. 1988;23(12):1204-9.

8. Cotton MF, Berkowitz FE, Ransome OJ. Candidal suppurative thrombophlebitis in a premature infant. Am J Dis Child. 1988;142(3):254-5.

9. Berg RA, Stein JM. Medical Management of Fungal Suppurative Thrombosis of great central veins in a child. Pediatr Infect Dis J. 1989;8(7):469-70.

10. Ashkenazi S, Pickering LK, Robinson LH. Diagnosis and Management of Septic Thrombosis of the inferior vena cava caused by Candida Tropicalis. Pediatr Infect Dis J. 1990;9(6):446-7.

11. Cherrick I, Kveselis DA, BaesI TJ. Medical Management of Candida-infected Thrombosis in a pediatric oncology patient. Pediatr Infect Dis J. 1995;14(5): 398-400.

12. Friedland IR. Peripheral thrombophlebitis caused by candida. Pediatr Infect Dis J. 1996;15(4):375-7. 
13. Pacifico L, Panero A, Colarizzi P, Matrunola M, Simonetti AF, Chiesa C. Neonatal Candida albicans Septic Thrombosis of the Portal Vein Followed by Cavernous Transformation of the Vessel. J Clin Microbiol. 2004;42(9):4379-82.

14. Sharma J, Nagraj A, Allapathi D, Rajegowda B. Leggiadro R fungal endocarditis in a premature infant complicated by a right atrial mycetoma and inferior vena cava thrombosis. Images Paediatr Cardiol. 2009;11(4):6-11.

15. Giuffrè M, Lo Verso C, Serrra G, Moceri G, Cimador M, Corsello G. Study Group of Neonatal Infectious Diseases Affiliated to the Italian Society of Neonatology. Portal vein thrombosis in a preterm newborn with mutation of the MTHFR and PAl-1 genes and Sepsis by Candida parapsilosis. Am J Perinatol. 2016;33(11):1099-103.

16. Arya MK, Ahmed SM, Shah KN, Ali US. Diabetic ketoacidosis with extreme hypernatremia in a 4-year-old girl Indian. J Crit Care Med. 2017;21(9):610-2.

17. Schapkaitz E, Weil R, White D, Klugman S. Asymptomatic catheter-related venous thrombosis in a child with cystic fibrosis: when to treat? J Vasc Nurs. 2019;37(1):43-5.

18. Torres-Rojas JR, Stratton CW, Sanders CV, Horsman TA, Hawley HB, Dascomb HE. Vial jr LJ. Candidal Suppurative peripheral thrombophlebitis. Ann Intern Med. 1982;96(4):431-5.

19. Garcia E, Granier I, Geissler A, Boespflug MD, Magnan PE, Durand-Gasselin J. Surgical management of Candida suppurative thrombophlebitis of superior vena cava after central venous catheterization. Intensive Care Med. 1997;23(9):1002-4.

20. Malfroot A, Verboven M, Levy J, Dab I, Naessens A, Delree M. Suppurative thrombophlebitis with Sepsis due to Candida Albicans: an unusual complication of intravenous therapy in cystic fibrosis. Pediatr Infect Dis. 1986;5(3):376-7.

21. Wisplinghoff $H$, Seifert $H$, Tallent SM, Bischoff T, Wenzel RP, Edmond MB, Nosocomial bloodstream infections in pediatric patients in United States hospitals: epidemiology, clinical features and susceptibilities. Pediatr Infect Dis J. 2003;22(8):686-91.

22. Chastre J, Cornud F, Bouchama A, Viau F, Benacerraf R, Gibert C. Thrombosis as a complication of pulmonary-artery catheterization via the internal jugular vein: prospective evaluation by phlebography. N Engl J Med. 1982;306(5):278-81.

23. Suetens C, Latour K, Kärki T, Ricchizzi E, Kinross P, Moro ML, Jans B, Hopkins S, Hansen S, Lyytikäinen O, Reilly J, Deptula A, Zingg W, Plachouras D, Monnet DL. "The Healthcare-Associated Infections Prevalence Study Group". Prevalence of healthcare-associated infections, estimated incidence and composite antimicrobial resistance index in acute care hospitals and longterm care facilities: results from two European point prevalence surveys, 2016 to 2017. Euro Surveill. 2018;23(46):1800516.

24. Nucci M, Anaissie E. Revisiting the source of candidemia: skin or gut? Clin Infect Dis. 2001;33(12):1959-67.

25. Colomba C, Siracusa L, Rubino R, Trizzino M, Scarlata F, Imburgia C, Titone L. A case of Brucella endocarditis in association with subclavian artery thrombosis. Case Rep Infect Dis. 2012;2012:581489.

26. Delfino D, Scordino F, Pernice I, Lo Passo C, Galbo R, David A, Barberi I, Criseo G, Cascio A, Romeo O. Potential association of specific Candida parapsilosis genotypes, bloodstream infections and colonization of health workers' hands. Clin Microbiol Infect. 2014;20(11):0946-51.

27. Walsh TJ, Rex JH. All catheter-related candidemia is not the same: assessment of the balance between the risks and benefits of removal of vascular catheters. Clin Infect Dis. 2002;34(5):600-2.

28. Chen K, Agarwal A, Tassone MC, Shahjahan N, Walton M, Chan A, Mondal T. Risk factors for central venous catheter-related thrombosis in children: a retrospective analysis. Blood Coagul Fibrinolysis. 2016;27(4):384-8.

29. Male C, Julian JA, Massicotle P, Gent M, Mitchell L. Protekt study group. Significant association with location of central venous line placement and risk of venous thrombosis in children. Thromb Haemost. 2005;94(3):516-21.

30. Romeo O, Delfino D, Cascio A, Lo Passo C, Amorini M, Romeo D, Pernice I. Microsatellite-based genotyping of Candida parapsilosis sensu stricto isolates reveals dominance and persistence of a particular epidemiological clone among neonatal intensive care unit patients. Infect Genet Evol. 2013;13:105-8.

31. Cascio A, Pantaleo D, Corona G, Barberi G, Delfino D, Romeo O, laria C, Barberi I. Neonatal liver abscesses associated with candidemia: three cases and review of literature. J Matern Fetal Neonatal Med. 2014;27(7):743-9.

32. Colomba C, Trizzino M, Imburgia C, Madonia S, Siracusa L, Giammanco GM. Candida glabrata meningitis and endocarditis: a late severe complication of candidemia. Int J Infect Dis. 2014;29:174-5.

\section{Publisher's Note}

Springer Nature remains neutral with regard to jurisdictional claims in published maps and institutional affiliations.

Ready to submit your research? Choose BMC and benefit from:

- fast, convenient online submission

- thorough peer review by experienced researchers in your field

- rapid publication on acceptance

- support for research data, including large and complex data types

- gold Open Access which fosters wider collaboration and increased citations

- maximum visibility for your research: over $100 \mathrm{M}$ website views per year

At BMC, research is always in progress.

Learn more biomedcentral.com/submissions 